\title{
Integrated Near Field Scaning System for High Frequency Deivces
}

\author{
Hengxu Li ${ }^{\# 1}$, Boyuan $\mathrm{Zhu}^{\# 2}$ \\ Queensland Micro- and Nanotechnology Centre \\ Griffith University \\ Brisbane, Australia \\ ${ }^{\# 1}$ jeffrey.li@griffithuni.edu.au \\ \#2boyuan.zhu@griffithuni.edu.au
}

\author{
M.Vatankhah Varnoosfadeani" ${ }^{\# 3}$, Junwei $\mathrm{Lu}^{\# 4}$ \\ Queensland Micro- and Nanotechnology Centre \\ Griffith University \\ Brisbane, Australia \\ \#3mohammad.vatankhahvarnoosfaderani@griffithuni.edu.au \\ ${ }^{\# 4}$ j.lu@griffith.edu.au
}

\begin{abstract}
In electromagnetic interference (EMI) applications, localization of real EMI sources in the near-field region is always in concern. This paper introduces an integrated near-field scanning system (INFSS) that handles electromagnetic near-field measurement of arbitrary 3D shape of high frequency devices in conjunction with numerical modelling and simulation results. It performs both finite element method (FEM) simulation and 3D near-field scanning function that help engineer to increase the project development efficiency and reduce the development time cost. A case study of a dual mode switch parasitic antenna is presented from design to measurement using INFSS.
\end{abstract}

Keywords- near-field; 3D scanning; integrated system; FEM simulation.

\section{INTRODUCTION}

Electromagnetic compatibility (EMC) is a branch of electrical research and science, which studies the electromagnetic power generation by the electronic product [1]. It is necessary for every electronic product to pass the EMC testing and make sure its energy emission does not affect the others or being affected. Within EMC studies, electromagnetic near field is more complicated than far field due to the electromagnetic field physic feature. Signals in near field have strong strength, large magnitude, fast variation and chaotic distribution [2]. It is critically important to investigate product electromagnetic features in near field in order to protect and improve the compatibility of the device in the EMI environment.

In EMI applications, numerical modelling and simulation offer a preview of electromagnetic field distribution of a device under test (DUT) in the early stage. To determine a spatial distribution of an electrical quantity in near-field region requests a measurement system, near-field scanner, to focus on localization of real EMI sources distributed in the highly reactive region surface of a DUT. Due to the irregular shape of different DUTs, it is sometime very difficult to access accurate position of point of interest [3].

In this paper, an INFSS is therefore presented. It is an integrated simulation and testing platform that performs numerical modelling, simulation and near-field measurement.
The 3D scanner is more capable of handling complicated structures. It directly converts the geometric CAD model created at design phase into $3 \mathrm{D}$ surface scanning path. The whole scanning process is automatically controlled by precise positioning motors with accuracy of 20 micros. Moreover, the testing result will be visualized and displayed on the computer. Thus, the engineer can conveniently compared the simulation result and 3D visualization near-field testing result. The following sections provide detailed descriptions of INFSS and a case study of a dual mode switch parasitic antenna which is used to prove the system reliability by comparing simulation result and 3D near-field scanning result.

\section{INFSS FEATURE AND STRUCTURE}

\section{A. INFSS}

INFSS is composed by two main components, the system flow chart is shown in figure 1. The basic concept of INFSS is to implement a geometric product modelling file in both FEM simulation and $3 \mathrm{D}$ near-field scanning. Product geometrical model file is generated by CAD software. It provides structural information such as product dimension and product surface mesh.

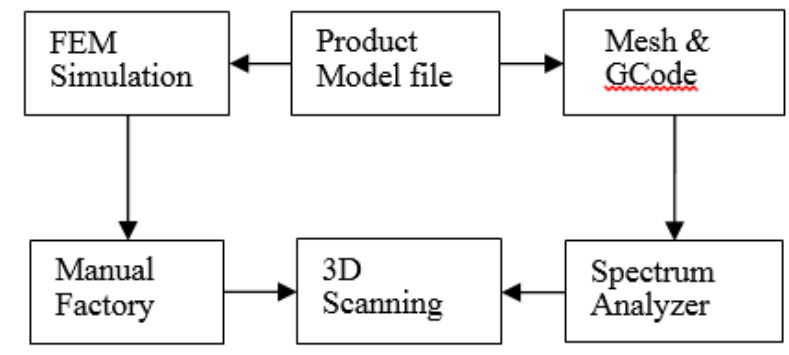

Fig. 1 INFSS Flow Chart

The first step to implement modelling file is to import it into FEM simulation software. The software will start computing to analyse the electric-field of product. By analyzed the FEM result, the engineer will predict the product performance and revise the product structure. The final product modelling file will be send to the manual factory to build the 
production prototype. The prototype will be send back to the laboratory for the further near-field investigation.

On the other hand, the previous CAD file also will be used to generate the mesh file. The mesh file will contain the testing point coordination information on the product surface. These information can be used in 3D scanner to control the movement of motor. The spectrum analyzer will be used to do the sampling and measurement in real time. The data will be collected and sent into the computer through USB connection for the analysis and result visualization purpose. The whole system structure is shown in figure 2 .

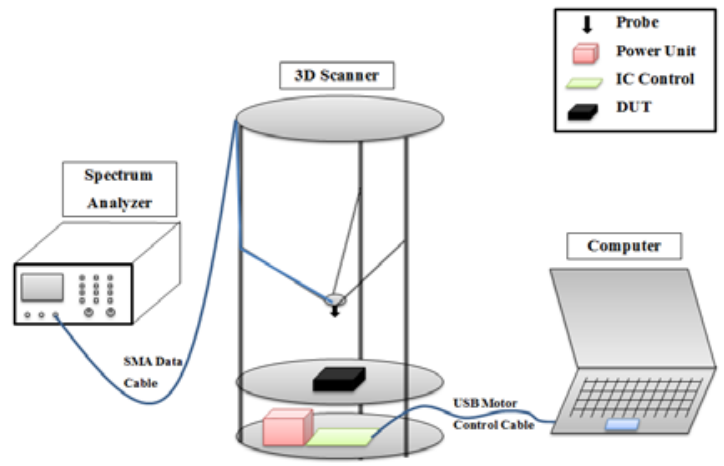

Fig. 2 INFSS Structure

\section{B. Mesh and GCode}

The mesh file contains a polygonal or polyhedral mesh that approximates a geometric domain [4]. The research implement the FEM package to generate the mesh information. The entire mesh will be applied in the FEM simulation. In the meantime, the surface mesh information can be used for the generation of GCode (as listed in table 1) which is the control command of 3D scanner. Based on mesh information, the 3D scanner will control the motor and move the probe on the product surface to measure the near-field electric-field line by line, which is shown in figure 3 .

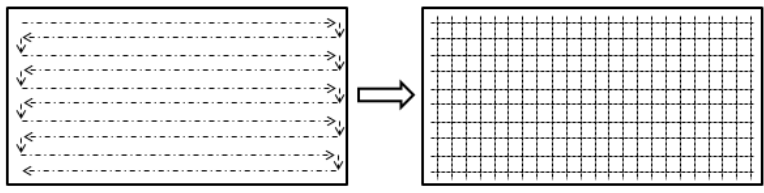

- Move

- Measure

Fig. 3 Mesh Generation and Motor Control

Table 1 GCode Structure

\begin{tabular}{|l|l|}
\hline Code & Descritpion \\
\hline $\mathrm{G} 1$ & Motor movement control code \\
\hline $\mathrm{X}$ & The x plane cooridnation \\
\hline $\mathrm{Y}$ & The Y plane cooridnation \\
\hline $\mathrm{Z}$ & The $\mathrm{Z}$ plane cooridination \\
\hline
\end{tabular}

\section{FEM Simulation}

A full-wave electromagnetic solution, which solves electromagnetic problems with Maxwell's equations, is of great importance to high performance VLSI design in computational electromagnetics. With numerous fast numerical algorithms developed, the finite element method (FEM) is relatively efficient in finding approximate solutions of partial differential equations (PDE). The frequency domain vector wave equation for $\mathrm{E}$ field can be derived as:

$$
\nabla \times \frac{1}{\mu} \nabla \times \vec{E}+\sigma_{e} \omega \vec{E}+\omega^{2} \varepsilon \vec{E}=-j \omega \vec{J}
$$

where $\omega$ is the angular frequency, $\mathrm{J}$ is the source current density, $\sigma_{\mathrm{e}}$ is the effective conductivity, and $\mu$ and $\varepsilon$ are the permeability and permittivity of the problem space respectively.

\section{3D Scanner}

The concept for 3D scanner (as shown in figure 4) is to implement the numerical and geometrical model and slice the target object to multiple layers. By used stepping motor and employed the precise computer digital control, the motor can position accurately in a three-dimension space. The specification of 3D scanner can be found in table 2 .

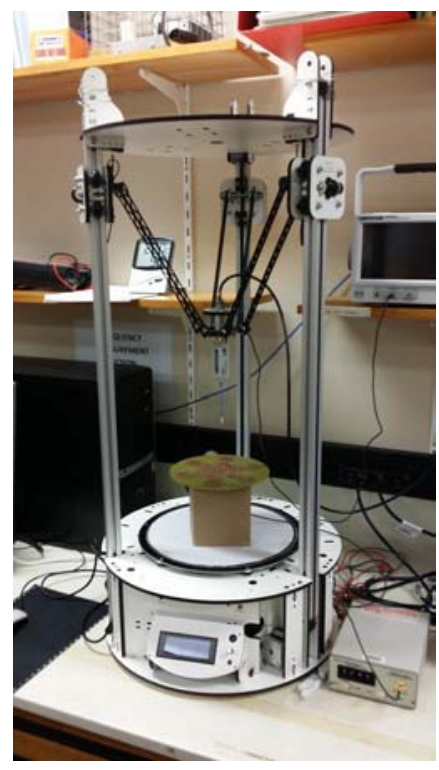

Fig. 4 3D scanner configuration

Table 2 3D Scanner Specification

\begin{tabular}{|l|l|}
\hline Diameter & $11^{\prime \prime}(27.9 \mathrm{~cm})$ \\
\hline Height & $143 / 4 '(37 \mathrm{~cm})$ \\
\hline Motor & NEMA 17 stepper \\
\hline Power supply unit & $450 \mathrm{~W}$ \\
\hline Position Accuracy & $0.02 \mathrm{~mm}(20$ micros $)$ \\
\hline Speed & $300 \mathrm{~mm} / \mathrm{s}$ \\
\hline
\end{tabular}




\section{CASe StUdy}

\section{A. Dual Mode Switch Parasitic Antenna}

The dual mode switch parasitic antenna is designed to satisfy the demands of the wearable wireless sensors and wireless body area networks communication. It is tuned to excite two desired modes at the same time to make patch resonate at $2.45 \mathrm{GHz}$ and $5 \mathrm{GHz}$, as shown in figure 5 .

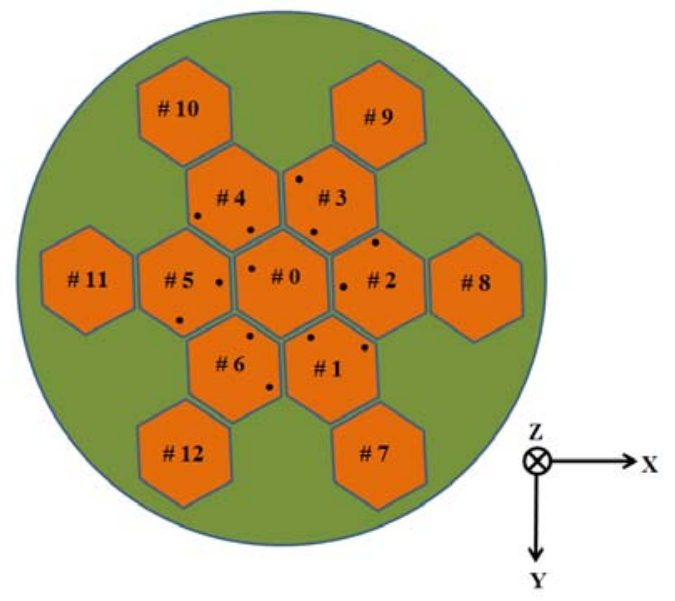

Fig. 5 Antenna Geometrical Structure

\section{B. Antenna Design}

Following Yagi-Uda antenna [5] directive and reflective parasitic elements are responsible to shape the radiation pattern in desired frequency. Central element $(\# 0)$ is a driven element surrounded by six hexagonal parasitic elements (\#1 - \#6) having equal size and shape. An additional six elements (\#7 \#12) were placed in an outer circle. These additional patches act as director elements to shape the radiation pattern and increase the directivity of the antenna in desired direction. The inner circle of six switched parasitic elements (or reactively loaded parasitic elements) remains in the same configuration as PASHE [6].Six switching parasitic elements were arranged around the main patch and can be open circuit or shorted to ground plane to sweep the pattern in desired direction using the switching circuit in figure.3. While the cavity model is unsuitable for detailed analysis of the PATHE antenna, but it provides a useful first order approximation for circular patch antenna.

$$
f_{(r) m n 0}=\frac{\chi_{m n}^{\prime}}{2 \pi a \sqrt{\mu \varepsilon}}
$$

While $f_{r}, \varepsilon, \mu$ and $\chi_{m n}^{\prime}$ are resonant frequency of the cavity, permittivity, permeability and zeros of derivatives of Bessel function. The dominant mode is $T M_{110}^{z}$ in circular patch antenna and $T M_{210}^{z}, T M_{010}^{z}$ and $T M_{310}^{z}$ are the next modes in ascending order [7]. The formula (2) does not include fringing effect and formula (3) and (4) are presented in [7] to make a correction for dominant mode.

$$
a=\frac{F}{\left\{1+\frac{2 h}{\pi \varepsilon_{r} F}\left[\ln \left(\frac{\pi F}{2 h}\right)+1.7726\right]\right\}^{1 / 2}}
$$

where

$$
F=\frac{8.791 \times 10^{9}}{f_{r} \sqrt{\varepsilon_{r}}}
$$

Parameter "a" is the radius of equivalent circular patch. Designing the dominant mode at $2.45 \mathrm{GHz}, T M_{010}^{z}$ mode would be excited at $5 \mathrm{GHz}$ because $\chi_{m n}^{\prime}$ is 1.8412 for $T M_{110}^{z}$ and 3.8318 for $T M_{010}^{z}$ which is proportional to $5 \mathrm{GHz}$ when 1.8412 is considered for $2.45 \mathrm{GHz}$. For a $1.6 \mathrm{~mm}$ FR4 epoxy substrate $\left(\varepsilon_{r}=4.5\right.$, tangent loss $\left.=0.025\right)$ and an operating frequency of $2.45 \mathrm{GHz}$, a $3.35 \mathrm{~cm}$ diameter patch was given. A parametric study has been done to find the best position for the feed to excite both $T M_{110}^{z}$ and $T M_{010}^{z}$ modes. Two orthogonal terminations were used for each of the switched elements. During operation, all outer ring elements (\#7- \#12) and one inner ring elements (e.g. \#1) function as director elements by switching to be ungrounded. The remaining elements in inner ring are short-circuited to ground at five directions in azimuth plane. Optimized dimensions of the main patch, the position of the feed and position of the shorting pins are depicted in figure 6 . The system consisted of four parts: the physical antenna, the switching circuitry, a USB controller and PC based software. A simple USB device was used to generate switching currents required for the antenna. A switching current of $4 \mathrm{~mA}$ at 4.8 volts was used to switch each element between open-circuit and short-circuit.

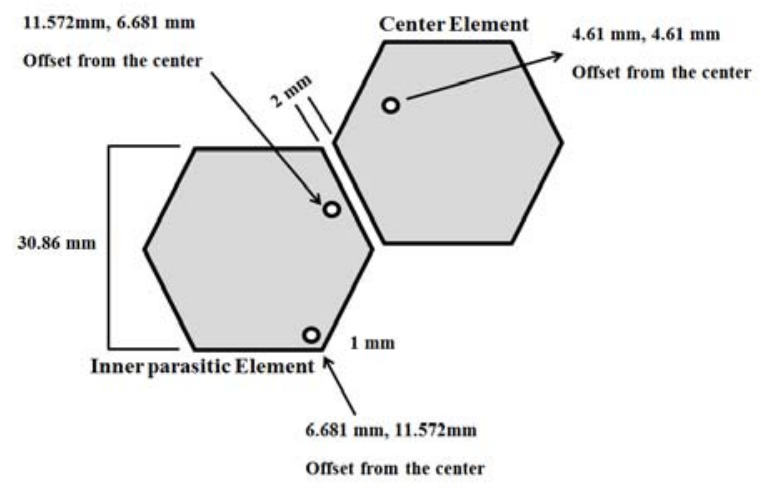

Fig. 6 Optimized Position of the Feed and Shorting Posts on the Patches

\section{Mesh Information for Antenna}

Based on the previous geometrical structure information, the antenna is sent into FEM package to generate the mesh information which is shown in the following figure 7 . The mesh method in this case is employing the Mapped mesh method and only mesh the product surface. 


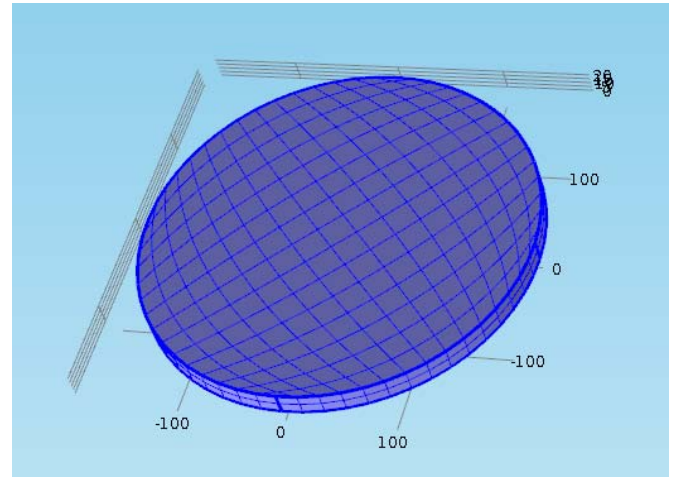

Fig. 7 Mesh Information for Antenna

\section{Result Comparison}

A ZVA24 ROHDE \& SCHWARZ network analyzer is used to measure the reflection coefficient of the antenna from 2 $\mathrm{GHz}$ to $6 \mathrm{GHz}$. Measurement results (as shown in figure 8) indicate that the $10 \mathrm{~dB}$ bandwidth of antenna is $200 \mathrm{MHz}$ $(8.3 \%)$ at $2.45 \mathrm{GHZ}$ and $320 \mathrm{MHz}$ at $5.15 \mathrm{GHz}(6.2 \%)$. The measured result is slightly shifted upward compared to simulated result and the explanation for that can be difference between the permittivity of the simulated FR4 and the real substrate.

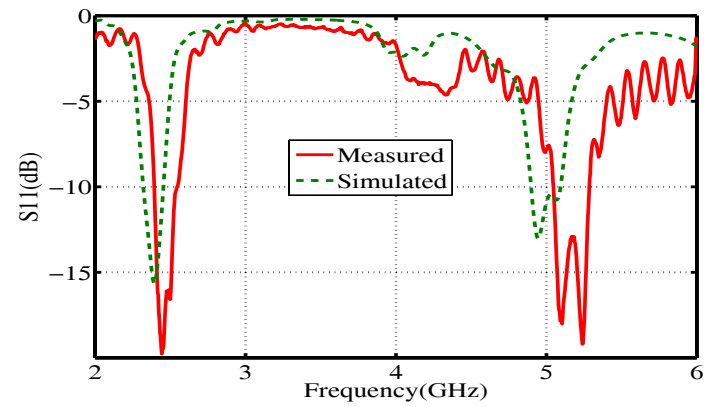

Fig. 8. Comparison of the simulated and measured return loss of the antenna

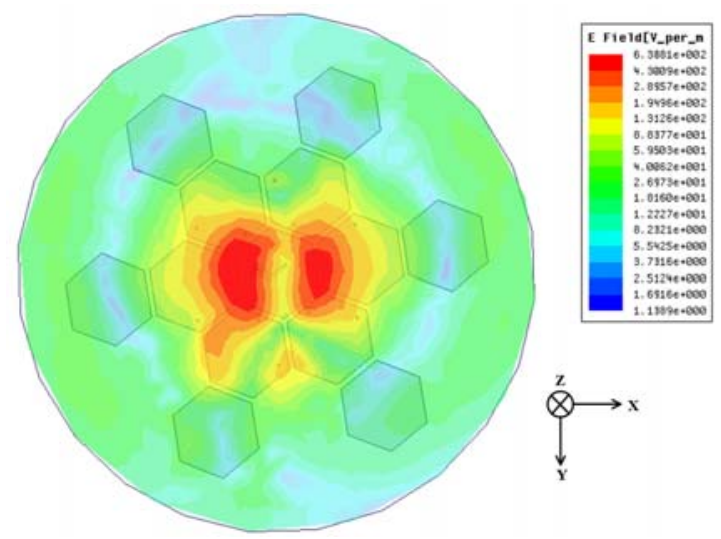

Fig. 9 Near-field Simulation Result

Therefore, near-field simulation and measurement are processed under corresponding resonant frequency as shown in figure 8 . The near-field simulation result and measurement are presented in figure 9 and 10 which give a very good agreement with each other. The color difference is due to different units presented.

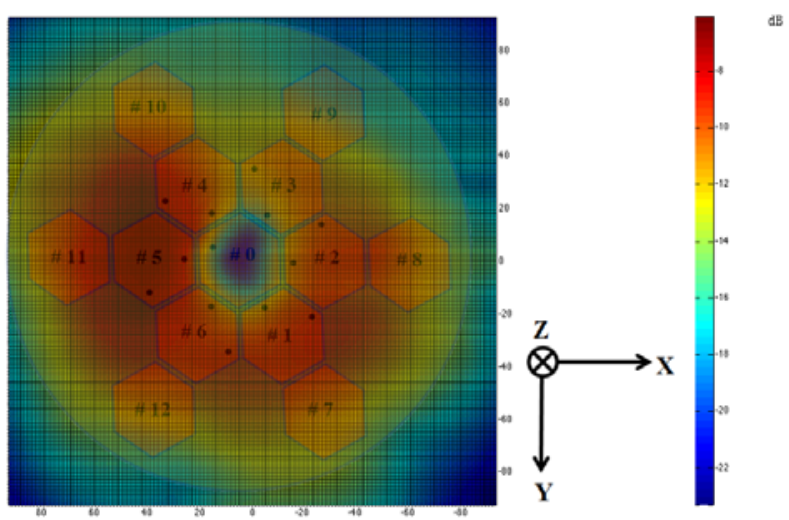

Figure 10 Near-field Measurement Result

\section{CONCLUSION}

In this paper, an integrated near-field scanning system has been presented for the high frequency device. The system implemented numerical modelling for both FEM simulation and 3D scanning. The system can help engineer obtain the accurately simulation result to analyse the product, and verify the design by automatically scanning the product's near field region. It significantly shortens project development time and increases efficiency.

\section{REFERENCES}

[1] C.R. Paul, "Introduction to electromagnetic compatibility," vol.184. John Wiley \& Sons, 2006.

[2] A.D.Yaghjian, "An overview of near-field antenna measurements," Antennas and Propagation, IEEE Transactions on , vol.34, no.1, pp.30,45, Jan 1986.

[3] W. Böhler \& A. Marbs, "3D scanning instruments," In Proceedings of the CIPA WG 6 International Workshop on Scanning for Cultural Heritage Recording, Ziti, Thessaloniki (pp. 9-18) 2002.

[4] H. Edelsbrunner, Geometry and topology for mesh generation. Cambridge University Press, 2001

[5] D. Gray, L. Jun Wei, and D. V. Thiel, "Electronically steerable YagiUda microstrip patch antenna array," in Antennas and Propagation Society International Symposium, 1995. AP-S. Digest, 1995, p. 1870 vol.4.

[6] W. H. Chen, J. W. Sun, X. Wang, Z. H. Feng, F. L. Chen, Y. Furuya, et al., "A Novel Planar Switched Parasitic Array Antenna With Steered Conical Pattern," Antennas and Propagation, IEEE Transactions on, vol. 55, pp. 1883-1887, 2007.

[7] C. A. Balanis, Antenna Theory - Analysis and Design (3rd Edition): John Wiley \& Sons, 2005.

[8] D. Gray, J.W. Lu \& D.V. Thiel, "Electronically steerable Yagi-Uda microstrip patch antenna array." Antennas and Propagation, IEEE Transactions on, 46(5), 605-608, 1998.

[9] W.H. Chen, J.W. Sun, X. Wang, X, Z.H. Feng, F.L. Chen, Y. Furuya \& A. Kuramoto, "A novel planar switched parasitic array antenna with steered conical pattern," Antennas and Propagation, IEEE Transactions on, 55(6), 1883-1887, 2007. 
